\title{
Radiation enhances the delivery of antisense oligonucleotides and improves chemo-radiation efficacy in brain tumor xenografts
}

\author{
Prakash Ambady ${ }^{1} \cdot$ Yingjen Jeffrey $\mathrm{Wu}^{1} \cdot$ Cymon N. Kersch ${ }^{1} \cdot$ Joshua M. Walker ${ }^{2,3} \cdot$ Samantha Holland ${ }^{1}$. \\ Leslie L. Muldoon ${ }^{1,2} \cdot$ Edward A. Neuwelt ${ }^{1,4,5}$
}

Received: 14 July 2020 / Revised: 25 January 2021 / Accepted: 16 March 2021 / Published online: 14 April 2021

(c) The Author(s) 2021. This article is published with open access

\begin{abstract}
Overexpression of $\mathrm{O}^{6}$-methylguanine DNA methyltransferase (MGMT) contributes to resistance to chemo-radiation therapy (CRT) in brain tumors. We previously demonstrated that non-ablative radiation improved delivery of anti-MGMT morpholino oligonucleotides (AMONs) to reduce MGMT levels in subcutaneous tumor xenografts. We evaluate this approach to enhance CRT efficacy in rat brain tumor xenograft models. The impact of radiation on targeted delivery was evaluated using fluorescent oligonucleotides (f-ON). In vitro, f-ON was localized to clathrin-coated vesicles, endosomes, and lysosomes using confocal microscopy in T98G glioma cells. In vivo, fluorescence was detected in pre-radiated, but not nonradiated Long Evans (non-tumor bearing) rat brains. Cranial radiation (2 Gy) followed by AMONs (intravenous, $10.5 \mathrm{mg} / \mathrm{kg}$ ) reduced MGMT expression by 50\% in both orthotopic cerebellar D283 medulloblastoma and intracerebral H460 non-small cell lung carcinoma (NSCLC) xenograft models. To evaluate the efficacy, AMONs concurrent with CRT (2 Gy radiation plus oral $20 \mathrm{mg} / \mathrm{kg}$ temozolomide $\times 4$ days) reduced tumor volumes in the medulloblastoma model $(p=0.012)$, and a similar trend was found in the NSCLC brain metastasis model. We provide proof of concept for the use of non-ablative radiation to guide and enhance the delivery of morpholino oligonucleotides into brain tumor xenograft models to reduce MGMT levels and improve CRT efficacy.
\end{abstract}

These authors contributed equally: Prakash Ambady, Yingjen Jeffrey $\mathrm{Wu}$

Supplementary information The online version contains supplementary material available at https://doi.org/10.1038/s41417021-00324-6.

Prakash Ambady

ambady@ohsu.edu

1 Department of Neurology, Oregon Health and Sciences University, Portland, OR, USA

2 Department of Cell, Developmental and Cancer Biology, Oregon Health and Science University, Portland, OR, USA

3 Department of Radiation Medicine, Oregon Health and Science University, Portland, OR, USA

4 Department of Neurosurgery, Oregon Health and Science University, Portland, OR, USA

5 Department of Veterans Affairs Medical Center, Office of Research and Development, Portland, OR, USA

\section{Introduction}

The addition of concurrent temozolomide to radiation therapy (chemo-radiotherapy; CRT) improves survival in patients with glioblastoma [1]. The cytotoxicity of temozolomide, an oral alkylating chemotherapy agent is primarily due to DNA methylation at the $\mathrm{O}^{6}$ position of guanine, triggering the DNA mismatch pathway leading to tumor cell apoptosis. $\mathrm{O}^{6}$-methylguanine DNA methyltransferase (MGMT) is a key DNA repair protein that can scavenge the methyl-groups from guanine and is associated with resistance to temozolomide [2-5]. MGMT is epigenetically inactivated by gene promoter methylation (methylated MGMT) in about $45 \%$ of patient-derived glioblastoma and MGMT gene methylation status remains the most significant predictive biomarker for better outcomes after CRT [6]. Thus, MGMT provides a promising therapeutic target for over half of glioblastoma patients that have unmethylated MGMT.

Morpholino antisense oligonucleotides are short synthetic nucleotide sequences that bind to complementary messenger RNA and block protein synthesis. They are 
specific, water-soluble, non-toxic, uncharged, and extremely stable under physiologic conditions, and are not cleaved by endogenous nucleases, thus providing an excellent platform for potential targeted therapies in oncology [7-9]. However, effective and targeted delivery of morpholino oligonucleotides to tumor sites is challenging, especially to the brain, where delivery of systemically administered agents across the neurovascular unit (NVU) remains a major hurdle [9-11]. We have previously reported the successful use of a non-ablative dose of ionizing radiation to prime human cancer cells to enhance the uptake of unmodified anti-MGMT morpholino oligonucleotides (AMONs) sequences to block MGMT mRNA translation [10]. In vitro, radiation-enhanced AMONs delivery decreased MGMT protein and significantly reduced human cancer cell proliferation index and cell viability in conjunction with temozolomide treatment [10]. In vivo, radiation enhanced the targeted delivery of a single intravenous dose of AMONs to achieve 50\% MGMT knockdown after 3 days. MGMT silencing only occurred in radiation-primed tumor sites in the subcutaneous tumor model. Targeted gene expression knockdown using morpholino antisense is rapidly advancing and shows significant translational potential based on recent FDA approvals [12, 13].

Fractionated ionizing radiation is already an integral part of brain tumor therapy and is known to transiently increase NVU permeability at early time points after exposure in a dose-dependent manner [14-17]. In this study, we evaluate the use of clinically relevant non-ablative doses of radiation to guide and enhance the delivery of intravenously administered AMONs to brain tumor sites to silence MGMT and enhance the efficacy of CRT in orthotopic rat models of MGMT-positive human brain tumors. Targeted silencing of MGMT in pre-radiated tumor sites using our novel approach, without measurable MGMT silencing in nonradiated sites, may limit systemic toxicities associated with prior approaches to deplete MGMT in combination with temozolomide [18-20].

\section{Materials and methods}

\section{Cell lines and reagents}

Human T98G glioma, H460 non-small cell lung carcinoma (NSCLC), and D283 medulloblastoma cell lines were obtained from American type culture collection (ATCC; Rockville, MD, USA) and were used at early passage numbers. Cells were cultured in recommended culture medium supplemented with $10 \%$ fetal bovine serum and $1 \%$ streptomycin/penicillin in a $5 \% \mathrm{CO}_{2}$ atmosphere at $37 \mathrm{C}$. A $3^{\prime}$-carboxyfluorescein labeled scrambled morpholino oligonucleotide (f-ON) was purchased from Gene Tools, LLC
(Philomath, OR, USA). Three unmodified anti-MGMT specific morpholino oligonucleotide sequences were obtained from Gene Tools and dosed as previously described [10]. The in vivo dosage of $\mathrm{f}-\mathrm{ON}$ and AMON $(10.5 \mathrm{mg} / \mathrm{kg})$ used in this study was calculated/converted from the manufacturer suggested in vitro effective dosage $(15 \mu \mathrm{M})$ of AMONs (molecular weight $8 \mathrm{kDa}$ ) with the assumption that the average blood volume in rats is around $10 \mathrm{ml}$. Temozolomide for oral gavage (Merck \& Co, New Jersey, USA) was purchased from Oregon Health and Science University (OHSU) pharmacy and diluted in sterile water to a concentration of $2.5 \mathrm{mg} / \mathrm{ml}$. Rabbit anti-MGMT (\#2739), poly-ADP polymerase (PARP; \#9532), early endosome antigen 1 (EEA1; \#3288), LAMP1 (\#9091), p27 kip1(\#2552), bcl-XL (\#2762) and clathrin (\#4796) antibodies was purchased from Cell Signaling Technology (Davers, MA, USA). Human mitochondrial antigen (HMT; ab 196611) was purchased from Abcam (Cambridge, MA) Mouse anti-tubulin antibody (T9026) was purchased from Sigma (St Louis, MO, USA).

\section{Western immunoblot}

Cancer cells or tissues were harvested at specified time points for each experiment. Whole-cell lysates were isolated and subjected to western blotting analysis as previously described [10]. Quantification of the immunoblotting signal of individual proteins was performed using the UN-SCANIT Gel software (Silk Scientific, Inc., Orem, UT, USA).

\section{In vitro f-ON uptake}

Cancer cells were exposed to radiation ( $2 \mathrm{~Gy}$ ) or no radiation (controls). Cells were incubated with f-ON $(15 \mu \mathrm{M})$ 1 day later and Texas red conjugated Wheat germ agglutinin (1:500) for $1 \mathrm{~h}$ at $37 \mathrm{C}$, then fixed in fresh $3.7 \%$ paraformaldehyde at room temperature for $15 \mathrm{~min}$ and washed with PBS. Membranes were not permeabilized. Immunohistochemistry was performed as described previously [21]. Images were collected the following day using a $60 \times / 1.4$ NA oil immersion objective on an inverted Zeiss LSM780 confocal microscope maintained by the OHSU Advanced Light Microscopy Core (Carl Zeiss, Oberkochen, German). Representative optical sections were collected using identical settings on the microscope and were analyzed using FIJI/ImageJ (NIH, Bethesda, MD, USA, https://fiji.sc) [22]. To further confirm this observation is dependent upon radiation dosing and incubation timing in D283 cells, we treated cells with $0,0.5,1$, and 2 Gy radiation and 1 day later cultured with f-ON $(15 \mu \mathrm{M})$ for 4 or $24 \mathrm{~h}$. Cells were then washed to remove free f-ON and then imaged using Axio Observer inverted microscope (Zeiss, Gottingen, Germany). 


\section{Brain irradiation}

The care and use of animals were approved by the institutional animal care and use committee and were under the supervision of the department of comparative medicine at OHSU. Rats were anesthetized with dexmedetomidine $(0.6 \mathrm{mg} / \mathrm{kg} \mathrm{IP})$ and ketamine $(15 \mathrm{mg} / \mathrm{kg})$ and confined in a lead container so only specific brain areas were irradiated while avoiding critical structures such as eyes and nasal cavity to minimize radiation treatment-related toxicity. A total dose of $2 \mathrm{~Gy}$ in tumor-bearing athymic rats or $5 \mathrm{~Gy}$ in normal Long Evan rats was randomly given in a single fraction using the RadSource RS2000 Irradiator at OHSU small animal research imaging core or the Versa HD (Elekta, Stockholm, Sweden) linear accelerator in the department of radiation oncology [10]. The same irradiators were used for all animals included in any particular experiment. The dose of radiation differed by tumor cell type and the optimal dose was determined in vitro for each cell line used. As previously described, non-ablative radiation is defined as radiation doses $<8$ Gy per fraction [11].

\section{Xenograft models}

Female nude $(r n u / r n u)$ rats $(200-250 \mathrm{~g})$ from the breeding colony maintained in the animal facility at OHSU were anesthetized with intraperitoneal ketamine $(60 \mathrm{mg} / \mathrm{kg})$ and diazepam $(7.5 \mathrm{mg} / \mathrm{kg})$. MGMT expressing cancer cells $\left(10^{6}\right.$ cells $/ 10 \mu \mathrm{L},>90 \%$ viability) were injected via a $2-\mathrm{mm}$ diameter hole drilled in the skull using a 27 -gauge needle at a rate of $1 \mu \mathrm{L} / \mathrm{min}$. H460 human NSCLC were implanted into the right caudate nucleus (bregma $=0$; lateral $0.31 \mathrm{~cm}$; vertical $-0.65 \mathrm{~cm}$ ) while the D283 medulloblastoma cells were injected into the cerebellum (bregma $=0$; posterior $=$ -1.1 , vertical $=-0.5$ ) in a stereotactic frame (David Kopf Instruments; Tujunga, CA).

\section{Fluorescent oligonucleotide delivery}

For assessment of f-ON delivery in normal rat brain, female Long Evan rats (200-250 g) were purchase from Charles River (Wilmington, MA). Long Evan rats received IV f-ON $(10.5 \mathrm{mg} / \mathrm{kg}$; IV) either alone $(n=3)$ or 1 day after $5 \mathrm{~Gy}$ whole-brain irradiation $(n=3)$. Delivery was also assessed in D283 medulloblastoma cerebellar xenografts 14 days after cerebellar implantation. Rats received f-ON alone $(n=2)$ or 1 day after 2 Gy brain irradiation $(n=3)$. Brains were harvested 1 day after $\mathrm{f}-\mathrm{ON}$ administration and sections were stained for human mitochnondrial antigen (HMT) as a marker of human tumor cells and ionized calcium-binding adapter molecule 1 (IBA1) as a marker of human tumor cells and microglia with Hoechst nuclear counterstain.

\section{AMON delivery}

The effect of radiation on AMON delivery to the brain was assessed in the D283 cerebellar tumor model $(n=14)$ 21 days after tumor implantation and the H460 NSCLC intracerebral xenograft model $(n=12) 5$ days after tumor implantation. Tumor-bearing rats received a single dose of 2 Gy radiation followed in 1 day with AMONs $(10.5 \mathrm{mg} / \mathrm{kg}$; IV) randomly given to half of the animals. Tumor-bearing cerebellum or brain hemisphere were harvested 3 days later. Relative MGMT protein expression was measured by immunoblotting of brain lysates and semi-quantified by immunoblotting signal intensity after normalized with tubulin protein.

\section{Tumor treatment studies}

The in vitro impact of AMONs on CRT efficacy was evaluated in the D283 cells. D283 cells were treated with 0 or $2 \mathrm{~Gy}$ radiation and 1 day later cultured with AMON $(15 \mu \mathrm{M})$ or saline for 2 days. Cells were then treated with temozolomide $(100 \mu \mathrm{g} / \mathrm{ml})$. One day after temozolomide treatment, the whole-cell lysate was collected for immunoblotting analysis and cell viability was determined using WST-1 reagent (Abcam, Cambridge, MA, USA), following the manufacturer's protocol. The effect of AMONs on CRT efficacy was evaluated in the D283 cerebellar tumor model $(n=14)$ beginning 21 days after tumor implantation and the H460 NSCLC intracerebral xenograft model $(n=$ 12) 5 days after tumor implantation. Tumor-bearing rats received a single dose of $2 \mathrm{~Gy}$ radiation plus four doses of oral temozolomide $(20 \mathrm{mg} / \mathrm{kg} \times 4$ days $)$. AMONs $(10.5 \mathrm{mg} / \mathrm{kg}$; IV) were randomly administered $24 \mathrm{~h}$ after radiation to half $(n=7)$ of animals in each model. Rats were euthanized 7 days after the start of CRT. Brains were excised, fixed in $10 \%$ buffered formalin, and egg-gelatin embedded for vibratome sectioning at $100 \mu \mathrm{m}$ in the coronal plane. Every fifth brain section was stained for HMT and then imaged at $35 \mu \mathrm{m}$ pixel diameter on an Epson 1640XL flatbed scanner using Adobe Photoshop software. Tumor volume was assessed using NIH ImageJ software by one of the authors (LLM) blinded to treatments as previously described [23].

\section{Statistical analysis}

For in vivo animal AMON delivery and tumor treatment studies, a sample size of $n=6$ is required to achieve a power of $85 \%$. All data are presented as mean \pm SEM. The Student $t$ test was used for the comparison of measurable variants between two groups. $P<0.05$ was considered statistically significant (GraphPad Prism 6.0; Graph Pad Software). 

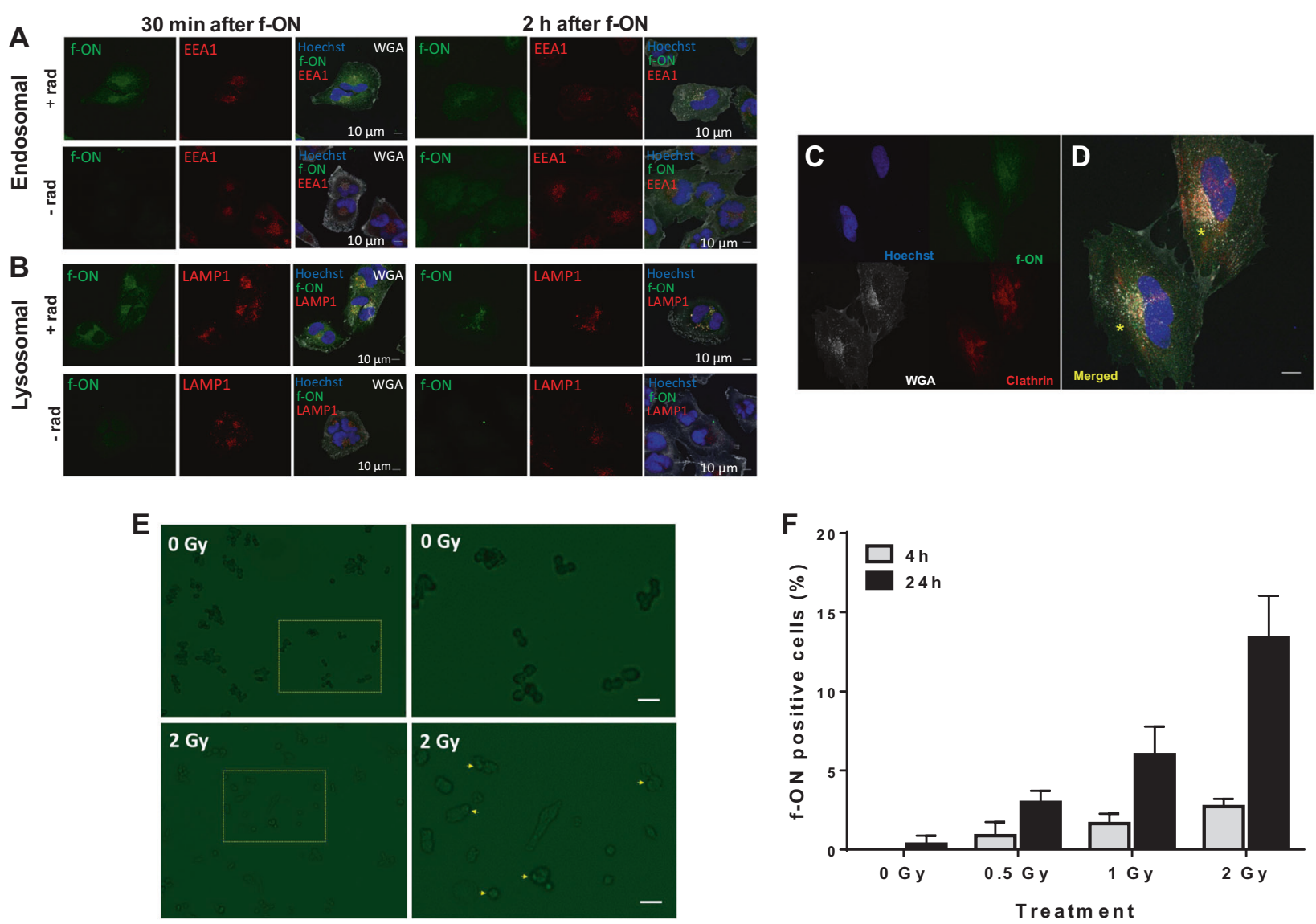

Fig. 1 Non-ablative radiation enhanced in vitro uptake of fluorescein-labeled scrambled oligonucleotides (f-ON) into endosomes and lysosomes. T98G cells were treated with $2 \mathrm{~Gy}$ radiation (top rows) or no radiation (bottom rows) 1 day prior to the addition of f-ON $(15 \mu \mathrm{M})$ for $30 \mathrm{~min}$ or $2 \mathrm{~h}$. Cells were washed and then fixed and immunostained for EEA1 (endosome marker, (A) or LAMP1 (lysosome marker, (B) with a counterstain for nuclei (Hoechst) and cell membrane (WGA). C, D Intracellular f-ON co-localize with areas of strong clathrin expression in T98G cells (1-day post $2 \mathrm{~Gy}$ ) after $30 \mathrm{~min}$ incubation with f-ON. E, F D283 cells were treated with $0,0.5,1$, and $2 \mathrm{~Gy}$ radiation and 1 day later incubated with $\mathrm{f}-\mathrm{ON}(15 \mu \mathrm{M})$ for 4 or

\section{Results}

\section{In vitro delivery of $\mathrm{f}-\mathrm{ON}$}

We assessed the impact of ionizing radiation ( $2 \mathrm{~Gy}$ ) on the intracellular delivery of $3^{\prime}$-carboxyfluorescein labeled nonspecific $\mathrm{f}-\mathrm{ON}$ administered 1 day after radiation in MGMTexpressing T98G glioma cells in vitro. Fluorescent signal was detected $30 \mathrm{~min}$ post $\mathrm{f}-\mathrm{ON}$ incubation in radiated cells but not in non-radiated cells. Intracellular f-ON co-localized with both the endosome marker EEA1 (Fig. 1A) and lysosome-associate membrane protein 1 (LAMP1, Fig. 1B) at the $30 \mathrm{~min}$ time point. At $2 \mathrm{~h}$ after f-ON administration, the endosomal fluorescence was no longer detected but the lysosomal localization remained strong (Fig. 1A, B). Intracellular f-ON co-localized with areas of strong clathrin
$24 \mathrm{~h}$. Cells were then washed to remove free f-AON and then imaged using Axio Observer inverted microscope (Zeiss, Gottingen, Germany). Bar scale $=15 \mu \mathrm{m}$. E Representative micrographs of cells given no-radiation (top row) and $2 \mathrm{~Gy}$ (bottom row) at $24 \mathrm{~h}$ after f-ON incubation, f-ON positive cells are indicated by the arrow. Micrographs in left and right panels were taken under $100 \mathrm{X}$ and $250 \mathrm{X}$ magnification, respectively. F Quantification of f-ON positive cells as a percentage. Data were presented as mean $\pm \mathrm{SD}$ of six randomly selected fields of view. Rad radiation, EEA early endosome antigen, LAMP lysosome-associated membrane protein, WGA wheat germ agglutinin.

expression (Fig. 1C, D). We confirmed radiation dose and time-dependent uptake of f-ON in D283 medulloblastoma cells (Fig. 1E, F). Compared to non-radiated control, $24 \mathrm{~h} \mathrm{f}$ ON incubation in combination with 2 Gy radiation showed the most $\mathrm{f}-\mathrm{ON}$ positive cells, with $13 \pm 2 \%$ of cells showing positive cell-associated fluorescent signal. These observations support our previous finding that radiation activated the endocytosis trafficking pathway in a dose-dependent manner to enhance morpholino intracellular delivery.

\section{In vivo delivery of $\mathrm{f}-\mathrm{ON}$ across the NVU}

We assessed the impact of cranial irradiation on the localization of $\mathrm{f}-\mathrm{ON}$ in the brain in non-tumor-bearing Long Evans rats and tumor-bearing athymic nude rats. No fluorescent signal was found in rat brains treated with either IV 

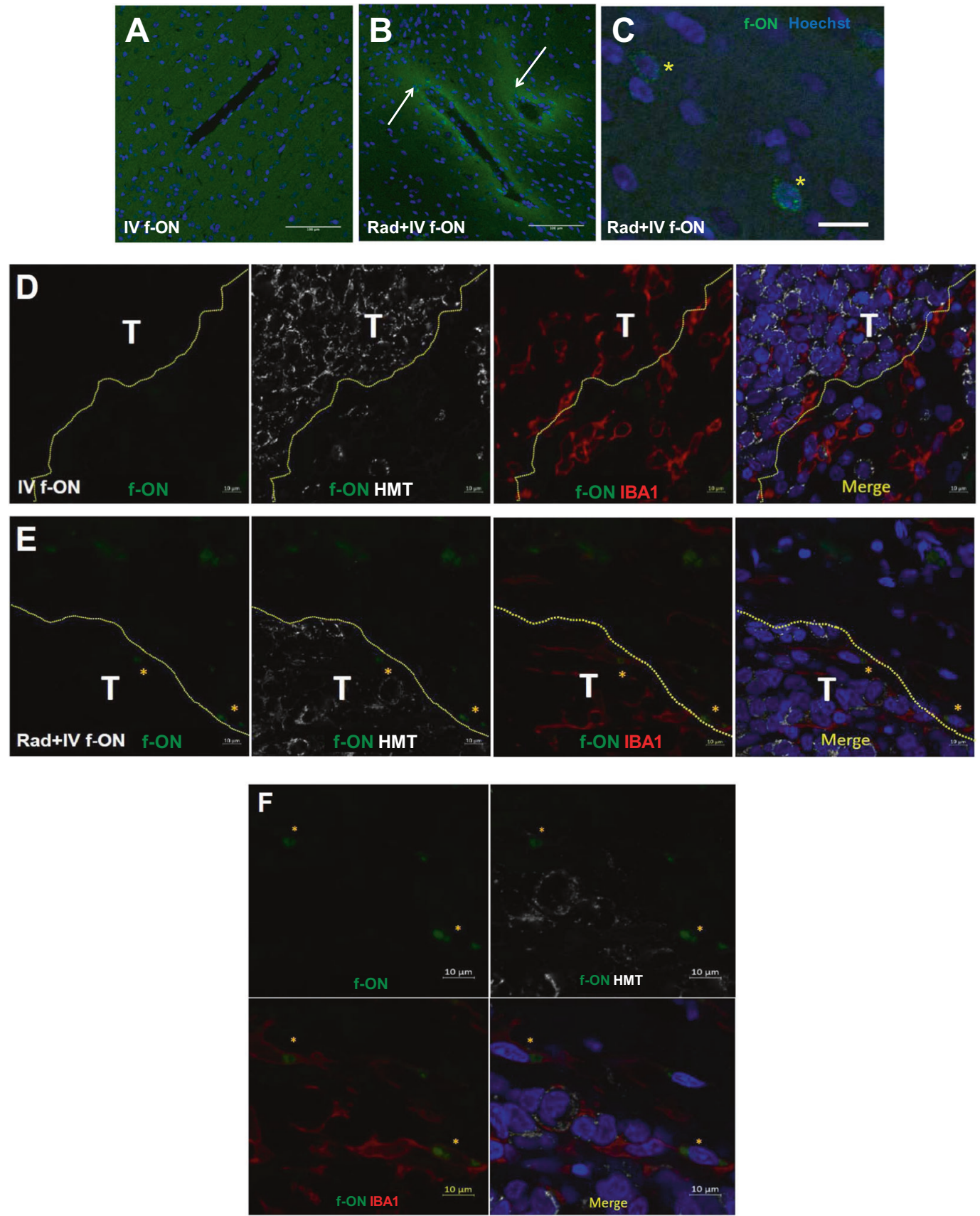

Fig. 2 Non-ablative radiation enhanced the delivery of f-ON into rat brain in vivo. A-C Long Evan rats received IV f-ON $(10.5 \mathrm{mg} / \mathrm{kg}$; IV) either alone (A) or 1 day after 5 Gy whole-brain irradiation (B), (C). Brains were harvested 1 day after f-ON administration. Sections were counterstained with Hoechst nuclear stain and fluorescein localization was analyzed. C High magnification field showing punctate cellular uptake of f-ON. D-F Athymic nude rats with D283 tumor received f-ON alone (D) or 1 day after 2 Gy brain irradiation observed;

f-ON alone without radiation (Fig. 2A). Non-tumor-bearing rats that received IV f-ON after radiation showed increased perivascular fluorescein signal in the brain (Fig. 2B). Under high magnification, parenchymal cells showed intracellular punctate fluorescence, suggesting the f-ON was taken up representative micrographs under low magnification $(\mathbf{E})$ or high magnification $(\mathbf{F})$. Brains were harvested 1 day after f-ON administration and sections were stained for human mitochnondrial antigen (HMT) and ionized calcium-binding adapter molecule 1 (IBA1) as a marker of human tumor cells and microglia. Scale bar $=100 \mu \mathrm{m}(\mathbf{A}$, B), $20 \mu \mathrm{m}(\mathbf{C})$, or $10 \mu \mathrm{m}(\mathbf{D}, \mathbf{E}, \mathbf{F})$, respectively. (T tumor and *representative f-ON positive cells).

into endosome and lysosome cellular compartments (Fig. 2C). In the absence of radiation, rats bearing cerebellar D283 medulloblastoma xenografts showed minimal f-ON fluorescent signal that was limited to the necrotic regions within the tumor and was not found in the brain around the 


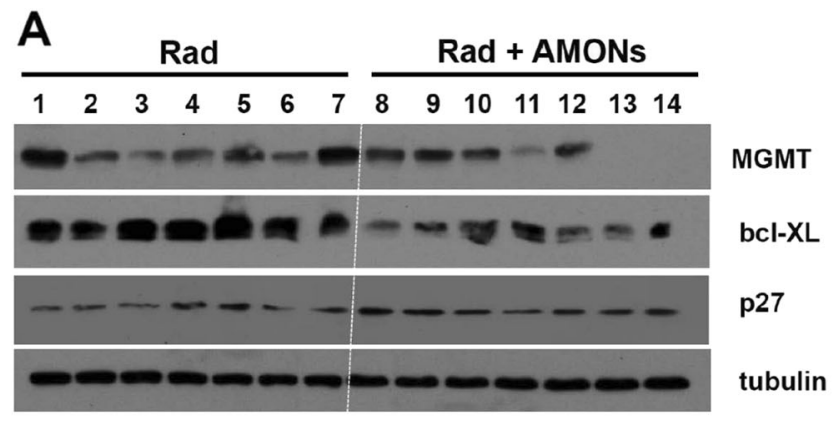

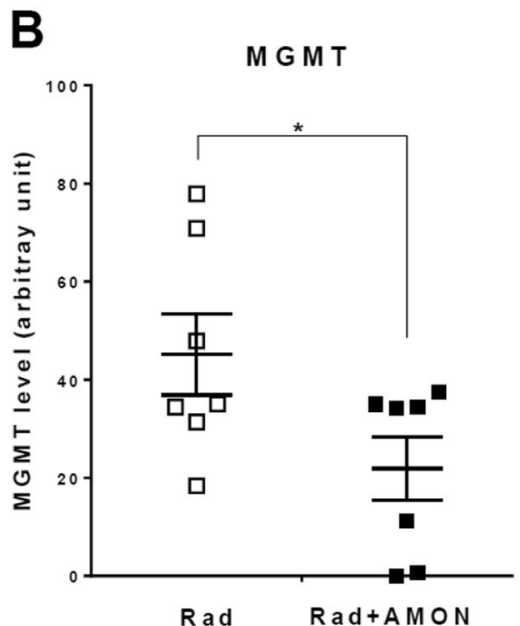

C

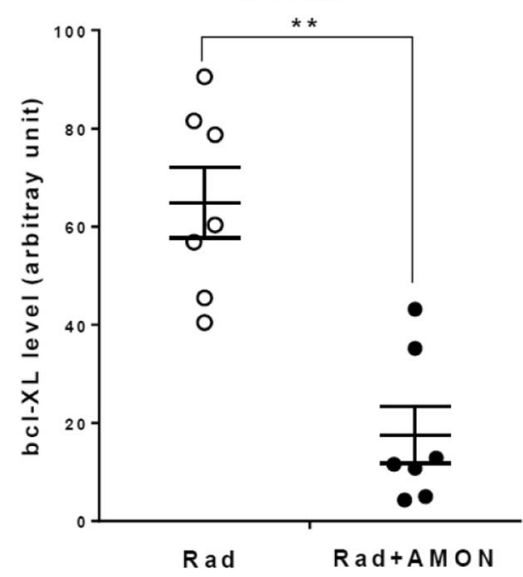

D

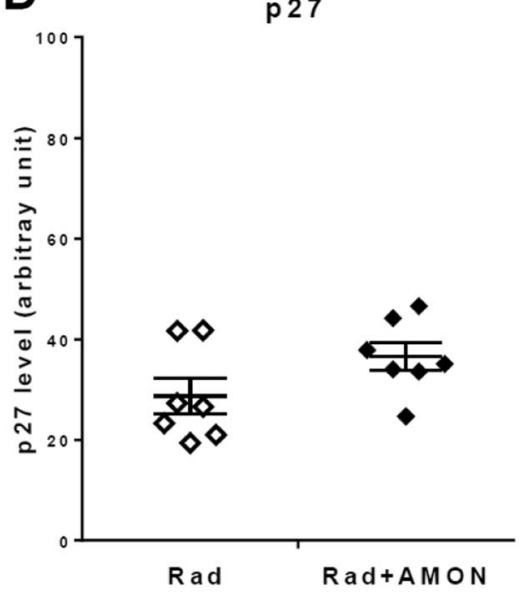

Fig. 3 Radiation-guided delivery of anti-MGMT oligonucleotides (AMONs) to reduce MGMT protein expression in D283 medulloblastoma orthotropic brain tumor model. Athymic nude rats were inoculated with MGMT expressing D283 medulloblastoma cells (intra-cerebellar, $n=14$ ). Rats received a single dose of $2 \mathrm{~Gy}$ cranial irradiation. AMON $(10.5 \mathrm{mg} / \mathrm{kg}$; IV) was administered 1 day after radiation to half $(n=7)$ of animals and all tumors were harvested

tumor (Fig. 2D). Fluorescent signal was increased in the irradiated tumors (Fig. 2E). Strong f-ON fluorescence was found predominantly but not limited to IBA1 positive cells in the brain around the tumor (Fig. 2F and Supplemental video). In addition, a diffuse parenchymal $\mathrm{f}-\mathrm{ON}$ signal was detected as far as $200 \mu \mathrm{m}$ from the tumor edge (Data not shown).

\section{Decreased MGMT by AMON in rat brain tumor xenografts}

In the D283 medulloblastoma orthotopic xenograft model, cranial radiation followed by IV AMON significantly decreased MGMT protein expression compared to radiation only ( $45.2 \pm 8.2$ vs. $21.9 \pm 6.5$ arbitrary unit, $p=0.04$; Fig. $3 \mathrm{~A}, \mathrm{~B})$. Compared to radiation alone, radiation plus AMONS led to a significant reduction $(64.9 \pm 7.2$ vs. $17.6 \pm 5.8, p<0.01)$ in the anti-apoptotic protein bcl-XL at 3 days (Fig. 3C). There was no difference in expression of p27, a biomarker used to confirm radiation exposure between these two groups (Fig. 3D). We found a non-
3 days later. A Immunoblot of MGMT, bcl-XL (apoptosis marker), and p27 (cell-cycle biomarker to confirm radiation delivery) of rat cerebellar tumors; Tubulin level was used as a total protein loading control. B-D Semi-quantification of MGMT, bcl-XL, and p27 immunoblotting signals. Data were presented as mean \pm SEM. Statistical significance indicated by $* P<0.05$. $* * P<0.01$.

statistically significant $(p>0.05)$ decrease in MGMT expression (35.7 \pm 16.1 vs. $13.3 \pm 8.2$; Supplemental Fig. $1 \mathrm{~A}, \mathrm{~B})$ and bcl-XL (41.3 \pm 9.3 vs. $23.3 \pm 5.8$; Supplemental Fig. 1C) expression comparing radiation alone to radiation plus AMON in H460 NSCLC xenografts (Supplemental Fig. 1).

\section{AMON enhances CRT efficacy in vitro of D283 cells and in MGMT expressing rat brain tumor xenografts}

Compared to traditional CRT ( $2 \mathrm{~Gy}+$ temozolomide), we demonstrated the addition of AMONs $(15 \mu \mathrm{M})$ at $24 \mathrm{~h}$ after 2 Gy radiation, significantly reduced D283 medulloblastoma cell viability (Supplemental Fig. 2A), and increase the presence of pro-apoptotic cleaved PARP protein (Supplemental Fig. 2B). These findings consistently agree with our previous publication. Furthermore, rats with orthotopic D283 medulloblastoma were treated with either CRT (radiation and temozolomide) or CRT with the addition of AMONs (experimental schema is shown in Fig. 4A). The optimal radiation dose and timing sequences were 

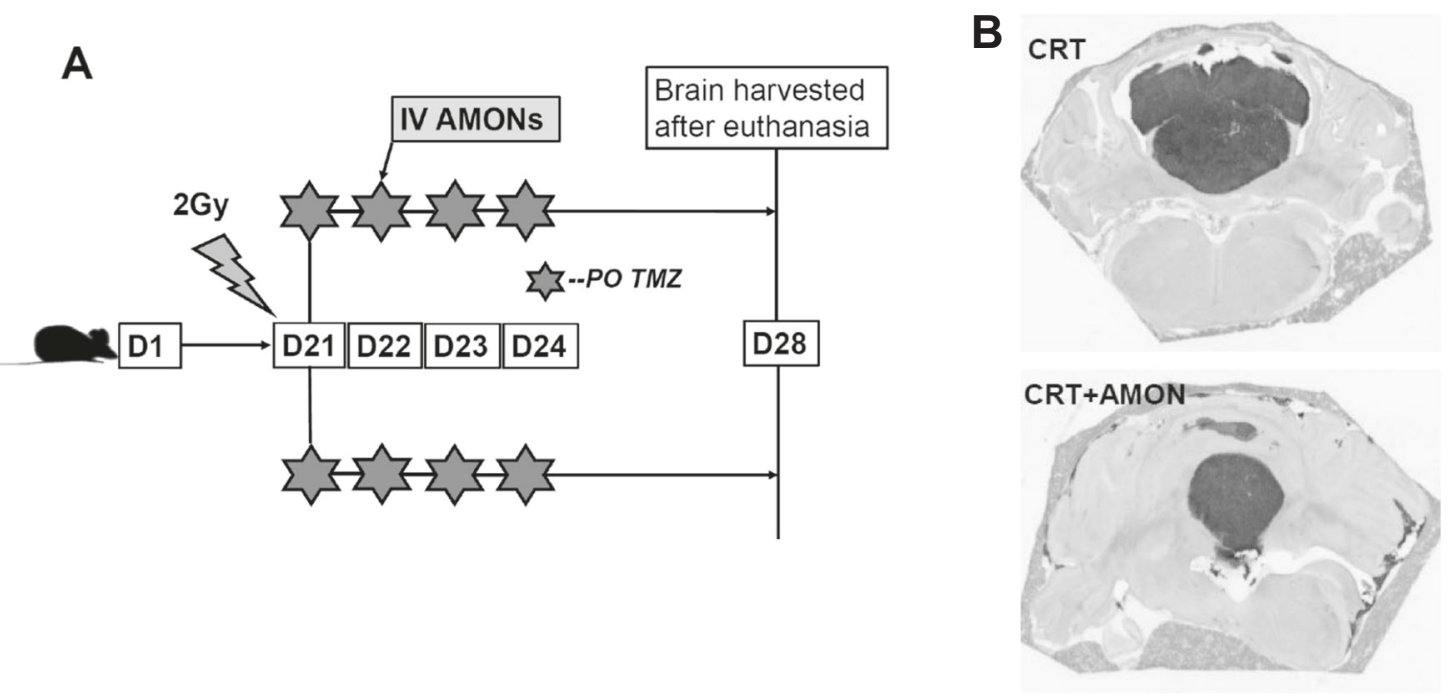

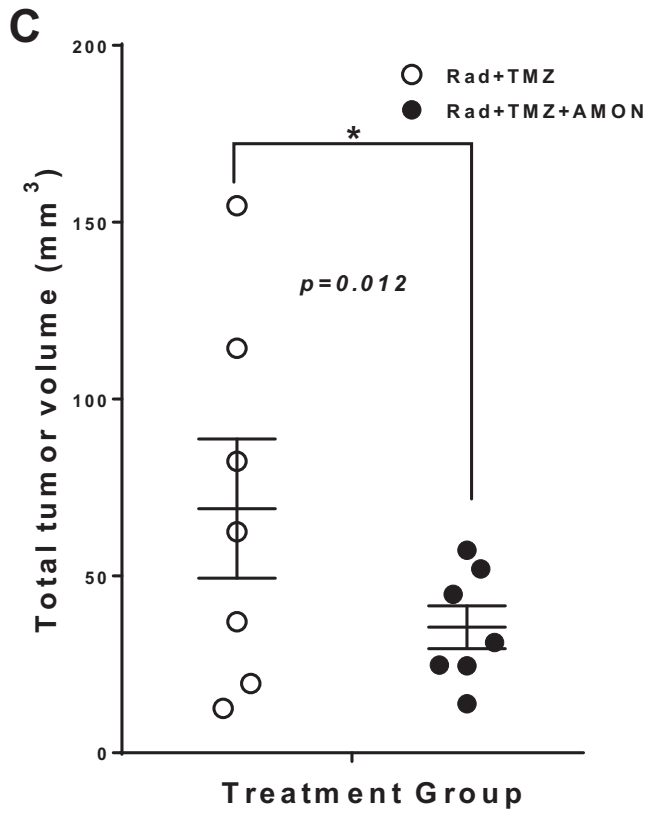

Fig. 4 AMON increased the efficacy of chemo-radiation therapy (CRT) in the D283 medulloblastoma orthotropic brain tumor model. Athymic nude rats were inoculated with MGMT expressing D283 medulloblastoma cerebellar orthotropic xenografts $(n=14)$. Tumor-bearing rats received a single dose of $2 \mathrm{~Gy}$ radiation with four daily doses of oral temozolomide $(20 \mathrm{mg} / \mathrm{kg} \times 4$ days $)$. AMONs $(10.5 \mathrm{mg} / \mathrm{kg}$; IV) were administered 1 day after radiation to half

determined from our prior studies [10]. Tumor volume was $69.0 \pm 19.7 \mathrm{~mm}^{3}$ after CRT only; addition of AMONs decreased tumor volume to $35.51 \pm 6.08 \mathrm{~mm}^{3}(p=0.012$; Fig. 4B, C). Four of seven animals in each group (57\%) showed disseminated leptomeningeal disease. In those animals that developed the leptomeningeal disease, AMON induced a $75 \%$ decrease of leptomeningeal tumor volume compared to CRT alone $\left(12.1 \pm 6.7\right.$ vs. $49.3 \pm 25.7 \mathrm{~mm}^{3}$; Fig. 4D), but the $p$-value was not significant ( $p=0.054$ ) due to the small sample size ( $n=4$ per group). No significant

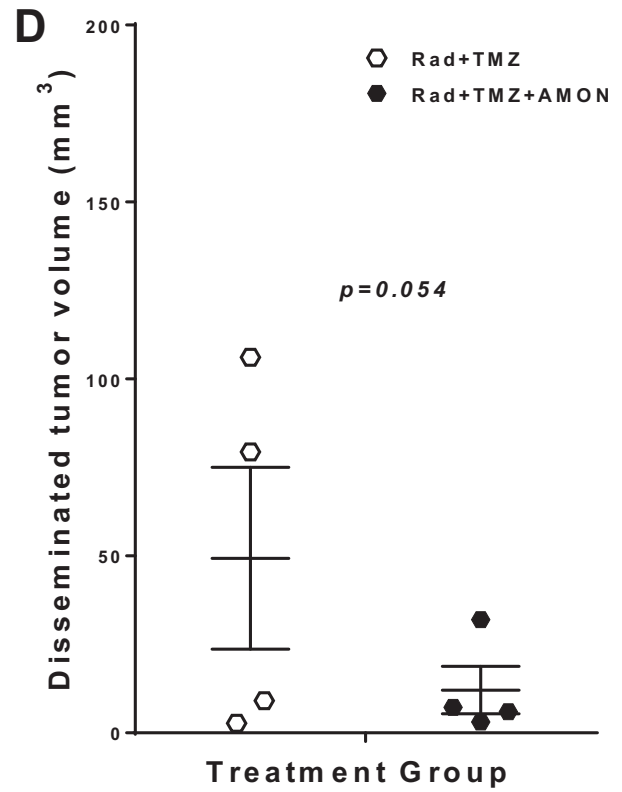

$(n=7)$ of animals and all tumors were harvested 7 days after the start of CRT. A Scheme of experimental design and treatment regime. B Representative after-treatment brain tumor histology from each treatment group. C Total tumor volumes and (D) disseminated tumor volumes are shown for individual animals. Data were presented as mean \pm SEM. Statistical significance indicated by $* P<0.05$. $* * P<$ 0.01 .

difference in percentage body weight loss was reported between AMON and CRT treatment groups $(3.8 \pm 1.5 \%$ vs. $2.8 \pm 1.2 \%$ ), suggesting that there were no additional systemic toxic effects of IV AMON administration compared to CRT alone. The effect of AMONs was also tested in the H460 NSCLC brain metastasis model (Supplemental Fig. 3). Although not statistically significant, we found a similar trend toward decreased tumor volume with the addition of AMONs, compared to CRT alone $(54.6 \pm 32.4$ vs. $91.4 \pm$ $38.9 \mathrm{~mm}^{3}, p=0.48$; Supplemental Fig. 3B, C). 


\section{Discussion}

The field of oligonucleotide therapeutics is rapidly evolving with the first antisense drug in the market and with promising agents in late-stage clinical trials [23-25]. Two major challenges in advancing oligonucleotide-based therapeutics for brain tumors including: (1) delivery of systemically administered oligonucleotides across the NVU and (2) convey the oligonucleotides to the correct intracellular compartment to achieve the desired effect within tumor tissues [11, 26, 27]. Unmodified morpholino oligonucleotides have very limited in vivo or in vitro ability to efficiently penetrate the eukaryotic plasma membrane without the aid of delivery strategies such as endo-porter or structural modifications [28]. The AMONs used in this study have 21-25 base pairs (molecular weight 7-9 kDa) and are expected to have very limited passive permeability across the NVU, even in enhancing brain tumors [29, 30]. Prior studies in rodent models have attempted to overcome the barrier of the NVU by using intra-ventricular or direct interstitial micro-perfusion of morpholinos to silence various target proteins in the CNS [31-33]. Drug administered into the cerebrospinal fluid is rapidly cleared into the blood via bulk flow through the CSF [34], while drug penetration from the CSF into brain parenchyma is poor and can range from as little as $0.3 \mathrm{~mm}$ for biologically active substances such as brain-derived neurotrophic factor to $2 \mathrm{~mm}$ for inert small molecules like hydroxyurea $[35,36]$. We have previously demonstrated the novel use of a non-ablative dose of radiation in combination with IV AMONs to achieve MGMT silencing in radiation primed tumors in a subcutaneous tumor model [10]. We have previously hypothesized that radiation may enhance the physiological dynamin-mediated endocytosis as demonstrated by the upregulation of caveolin 1 at $30 \mathrm{~min}$ after radiation, while clathrin and dynamin 2 expression peaked at $24-48 \mathrm{~h}$ after ionizing radiation. We adopted the radiation dose ( $2 \mathrm{~Gy}$ ) and timing $(24 \mathrm{~h})$ of AMON administration based on our prior publication [10]. Here (Fig. 1E, F), we further demonstrate the best intracellular delivery of f-ONs when D283 cells were given $24 \mathrm{~h}$ after $2 \mathrm{~Gy}$ radiation. Confocal fractionated cranial radiation (1.8-2 Gy) delivered over 6 weeks is the standard-of-care treatment for glioblastomas and other primary brain tumors including medulloblastoma [1]. Thus the choice of $2 \mathrm{~Gy}$ for our experiments provides additional clinical and translational significance.

Preclinical and clinical evidence suggests that in addition to direct DNA damage, radiation therapy can induce transient focal NVU disruption $[37,38]$. We found that radiation enhanced the delivery of f-ON across the NVU in normal and tumor-bearing rats, and radiation-induced physiologic endocytosis plays a key role in the intracellular delivery of morpholinos. MGMT was used as a prototypic protein and temozolomide as chemotherapy in this proof of concept study. We found that radiation-enhanced delivery of AMONs blocks MGMT synthesis by interfering with the translation of MGMT mRNA and specifically decreased MGMT expression in intracerebral tumors. Thus, the use of carefully contoured delivery of non-ablative radiation dose fractions holds the translational potential to enhance the delivery of AMONs in the targeted field without significant uptake in non-radiated sites. Radiation exposure is well known to enhance the expression of key proteins in the physiological endocytosis pathway, possibly mediating intracellular delivery of morpholino oligomers. This study provides additional evidence to support the hypothesis that once they cross the NVU, morpholino oligomers are taken up into the intracellular compartment through the endocytic pathway as evident by fluorescent signal localized to clathrin-coated vesicles, endosomes, and lysosomes using confocal microscopy (Fig. 1). Further, we demonstrate in vivo fluorescent signal in pre-radiated non-tumor bearing rat brain after intravenous administration of $\mathrm{f}-\mathrm{ONs}$ but not in non-radiated rat brains (Fig. 2). Our observations suggest that radiation-enhanced delivery may be beneficial in silencing target proteins in both radiologically nonenhancing as well as enhancing brain tumors within the contours of the radiation field. Targeted radiation-guided delivery limits off-target pharmacodynamic effects, bone marrow, and other systemic toxicities frequently seen with alternative approaches using MGMT pseudo-substrates such as $\mathrm{O}^{6}$-benzylguanine and $\mathrm{O}^{6}$-(4-bromothenyl) guanine [39-41]. No significant difference in percentage body weight loss was reported between AMON and CRT treatment groups in our study.

The role of MGMT is most relevant in the management of glioblastoma. One major limitation of the current studies is the lack of MGMT-expressing glioblastoma xenograft models. We have tested multiple commercially available and patient-derived un-methylated MGMT glioblastoma lines from our collaborators but failed to form MGMT protein-expressing brain tumors after orthotropic inoculation in athymic rats (data not shown). We hypothesize that this may reflect an epigenetic change in the in vivo tumor methylation status after implantation. Further, it is well known that there is a discordance between MGMT methylation as measured by methylation-specific polymerase chain reaction and MGMT mRNA levels and protein expression [42]. Hence, we used established H460 NSCLC and D283 medulloblastoma xenografts that continue to express MGMT in nude rat brains. Both cancer cell lines are traditionally resistant to alkylating agents potentially due to high levels of MGMT protein expression. In contrast to D283, H460 cells are more resistant to radiation. In addition, we also noted a large intertumoral heterogeneity in tumor volumes, this may have impacted the lack of 
statistical significance in our H460 model, even though we noted a strong trend toward decreased MGMT and increased CRT efficacy by AMONs. The target protein silencing achieved with morpholino oligomers is transient (3-7 days) [11]. However, the current standard of care using daily dose ( $2 \mathrm{~Gy}$ ) fractions of radiation over 6 weeks provides a window of opportunity for a potential daily AMON dosing schedule and sustained MGMT silencing over this time period to enhance the efficacy of CRT in unmethylated for high-grade glioma and potentially other primary brain tumors such as medulloblastoma [2, 43].

In conclusion, we demonstrated that compared to CRT alone, the addition of a single dose IV AMON combined with CRT significantly enhanced CRT efficacy in an MGMT expressing xenograft brain tumor model. We provide hypothesis-generating data to support further evaluation of IV AMONs in combination with standard CRT regimes to enhance its efficacy in MGMT overexpressing brain tumors. The dosage, frequency, and timing of AMON and optimal radiation dose fractions to achieve optimal efficacy needs further investigated and defined in future small animal and early phases in human studies. This study also supports further exploration of our approach to silence alternate clinically relevant target cancer-related proteins to enhance the efficacy of CRT.

Acknowledgements The authors would also like to thank Mr. Mike Pagel and Ms. Jessica Bills for their excellent technical support and Ms. Sofia Gallamore for her administrative support.

Funding This work was supported by OHSU Knight Cancer Institute Hillcrest Committee Pilot Project Award to PA; and the National Institutes of Health National Cancer Institute grants CA137488, CA199111, a Veterans Administration Merit Review grant, Jonathan D. Lewis Foundation Scholars Grant, and the Walter S. and Lucienne Driskill Foundation, all to EAN.

\section{Compliance with ethical standards}

Conflict of interest All morpholino oligonucleotides used were synthesized as per authors' specifications and purchased, except the AMON sequences that were provided free of cost by Gene Tools, LLC, (Philomath, OR, USA). The US patent application (\#16/ 522,381 ) entitled "morpholino oligonucleotides useful in cancer treatment" was issued to authors PA, YJW and EAN by the US patent office. Other authors have no financial interests or a conflict of interest with the results reported in this manuscript.

Publisher's note Springer Nature remains neutral with regard to jurisdictional claims in published maps and institutional affiliations.

Open Access This article is licensed under a Creative Commons Attribution 4.0 International License, which permits use, sharing, adaptation, distribution and reproduction in any medium or format, as long as you give appropriate credit to the original author(s) and the source, provide a link to the Creative Commons license, and indicate if changes were made. The images or other third party material in this article are included in the article's Creative Commons license, unless indicated otherwise in a credit line to the material. If material is not included in the article's Creative Commons license and your intended use is not permitted by statutory regulation or exceeds the permitted use, you will need to obtain permission directly from the copyright holder. To view a copy of this license, visit http://creativecommons. org/licenses/by/4.0/.

\section{References}

1. Stupp R, Mason WP, van den Bent MJ, Weller M, Fisher B, Taphoorn MJ, et al. Radiotherapy plus concomitant and adjuvant temozolomide for glioblastoma. $N$ Engl J Med. 2005;352:987-96.

2. Yu W, Zhang L, Wei Q, Shao A. O(6)-methylguanine-DNA methyltransferase (MGMT): challenges and new opportunities in glioma chemotherapy. Front Oncol. 2019;9:1547.

3. Bobola MS, Berger MS, Silber JR. Contribution of O6methylguanine-DNA methyltransferase to resistance to 1,3-(2chloroethyl)-1-nitrosourea in human brain tumor-derived cell lines. Mol Carcinog. 1995;13:81-8.

4. Carlson BL, Grogan PT, Mladek AC, Schroeder MA, Kitange GJ, Decker PA, et al. Radiosensitizing effects of temozolomide observed in vivo only in a subset of O6-methylguanine-DNA methyltransferase methylated glioblastoma multiforme xenografts. Int J Radiat Oncol Biol Phys. 2009;75:212-9.

5. Jiang G, Jiang AJ, Xin Y, Li LT, Cheng Q, Zheng JN. Progression of $\mathrm{O}(6)$-methylguanine-DNA methyltransferase and temozolomide resistance in cancer research. Mol Biol Rep. 2014;41:6659-65.

6. Hegi ME, Diserens AC, Gorlia T, Hamou MF, de Tribolet N, Weller M, et al. MGMT gene silencing and benefit from temozolomide in glioblastoma. N Engl J Med. 2005;352:997-1003.

7. Stirchak EP, Summerton JE, Weller DD. Uncharged stereoregular nucleic acid analogs: 2. Morpholino nucleoside oligomers with carbamate internucleoside linkages. Nucleic Acids Res. 1989;17:6129-41.

8. Summerton J, Weller D. Morpholino antisense oligomers: design, preparation, and properties. Antisense Nucleic Acid Drug Dev. 1997;7:187-95.

9. MacLeod AR, Crooke ST. RNA therapeutics in oncology: advances, challenges, and future directions. J Clin Pharmacol. 2017;57(Suppl 10):S43-S59.

10. Ambady P, Wu YJ, Walker JM, Kersch C, Pagel MA, Woltjer RL, et al. Enhancing the cytotoxicity of chemoradiation with radiationguided delivery of anti-MGMT morpholino oligonucleotides in non-methylated solid tumors. Cancer Gene Ther. 2017;24:348-57.

11. Puhalla S, Elmquist W, Freyer D, Kleinberg L, Adkins C, Lockman $\mathrm{P}$, et al. Unsanctifying the sanctuary: challenges and opportunities with brain metastases. Neuro Oncol. 2015;17:639-51.

12. Aartsma-Rus A, Krieg AM. FDA approves eteplirsen for duchenne muscular dystrophy: the next chapter in the eteplirsen saga. Nucleic Acid Ther. 2017;27:1-3.

13. Scoles DR, Pulst SM. Oligonucleotide therapeutics in neurodegenerative diseases. RNA Biol. 2018;15:707-14.

14. Trnovec T, Kallay Z, Bezek S. Effects of ionizing radiation on the blood brain barrier permeability to pharmacologically active substances. Int J Radiat Oncol Biol Phys. 1990;19:1581-7.

15. Fauquette W, Amourette C, Dehouck MP, Diserbo M. Radiationinduced blood-brain barrier damages: an in vitro study. Brain Res. 2012;1433:114-26.

16. Diserbo M, Agin A, Lamproglou I, Mauris J, Staali F, Multon E, et al. Blood-brain barrier permeability after gamma whole-body irradiation: an in vivo microdialysis study. Can J Physiol Pharm. 2002;80:670-8. 
17. Nakata H, Yoshimine T, Murasawa A, Kumura E, Harada K, Ushio Y, et al. Early blood-brain barrier disruption after high-dose single-fraction irradiation in rats. Acta Neurochir. 1995;136:82-6. ; discussion 6-7

18. Quinn JA, Jiang SX, Reardon DA, Desjardins A, Vredenburgh JJ, Rich JN, et al. Phase I trial of temozolomide plus $\mathrm{O}^{6}$-benzylguanine 5-day regimen with recurrent malignant glioma. Neuro Oncol. 2009; 11:556-61.

19. Broniscer A, Gururangan S, MacDonald TJ, Goldman S, Packer RJ, Stewart CF, et al. Phase I trial of single-dose temozolomide and continuous administration of $\mathrm{O}^{6}$-benzylguanine in children with brain tumors: a pediatric brain tumor consortium report. Clin Cancer Res. 2007;13:6712-8.

20. Weingart J, Grossman SA, Carson KA, Fisher JD, Delaney SM, Rosenblum ML, et al. Phase I trial of polifeprosan 20 with carmustine implant plus continuous infusion of intravenous $\mathrm{O}^{6}$-benzylguanine in adults with recurrent malignant glioma: new approaches to brain tumor therapy CNS consortium trial. J Clin Oncol. 2007;25:399-404.

21. $\mathrm{Wu}$ YJ, Neuwelt AJ, Muldoon LL, Neuwelt EA. Acetaminophen enhances cisplatin- and paclitaxel-mediated cytotoxicity to SKOV3 human ovarian carcinoma. Anticancer Res. 2013;33:2391-400.

22. Schneider CA, Rasband WS, Eliceiri KW. NIH Image to ImageJ: 25 years of image analysis. Nat Methods. 2012;9:671-5.

23. Wang L, Prakash RK, Stein CA, Koehn RK, Ruffner DE. Progress in the delivery of therapeutic oligonucleotides: organ/cellular distribution and targeted delivery of oligonucleotides in vivo. Antisense Nucleic Acid Drug Dev. 2003;13:169-89.

24. Asami Y, Yoshioka K, Nishina K, Nagata T, Yokota T. Drug delivery system of therapeutic oligonucleotides. Drug Disco Ther. 2016;10:256-62.

25. Juliano RL. The delivery of therapeutic oligonucleotides. Nucleic Acids Res. 2016;44:6518-48.

26. Naidoo J, Panday H, Jackson S, Grossman SA. Optimizing the delivery of antineoplastic therapies to the central nervous system. Oncology. 2016;30:953-62.

27. Neuwelt E, Ambady P, Muldoon L, McConnell H, Doolittle N. Outwitting the blood-brain barrier. Oncology. 2016;30:963

28. Morcos PA, Li Y, Jiang S. Vivo-Morpholinos: a non-peptide transporter delivers Morpholinos into a wide array of mouse tissues. Biotechniques. 2008;45:613-4. 6,passim

29. Pardridge WM. Drug transport across the blood-brain barrier. J Cereb Blood Flow Metab. 2012;32:1959-72.

30. Lockman PR, Mittapalli RK, Taskar KS, Rudraraju V, Gril B, Bohn KA, et al. Heterogeneous blood-tumor barrier permeability determines drug efficacy in experimental brain metastases of breast cancer. Clin Cancer Res. 2010;16:5664-78.
31. Oh IS, Shimizu H, Satoh T, Okada S, Adachi S, Inoue K, et al. Identification of nesfatin-1 as a satiety molecule in the hypothalamus. Nature. 2006;443:709-12.

32. Silvestre DC, Gil GA, Tomasini N, Bussolino DF, Caputto BL. Growth of peripheral and central nervous system tumors is supported by cytoplasmic c-Fos in humans and mice. PLoS ONE. 2010;5:e9544.

33. Oh IS, Shimizu H, Sato T, Uehara Y, Okada S, Mori M. Molecular mechanisms associated with leptin resistance: n-3 polyunsaturated fatty acids induce alterations in the tight junction of the brain. Cell Metab. 2005;1:331-41.

34. Pardridge WM. Drug transport in brain via the cerebrospinal fluid. Fluids Barriers CNS. 2011;8:7.

35. Mak M, Fung L, Strasser JF, Saltzman WM. Distribution of drugs following controlled delivery to the brain interstitium. J Neurooncol. 1995;26:91-102.

36. Blasberg RG, Patlak C, Fenstermacher JD. Intrathecal chemotherapy: brain tissue profiles after ventriculocisternal perfusion. J Pharm Exp Ther. 1975;195:73-83.

37. Qin DX, Zheng R, Tang J, Li JX, Hu YH. Influence of radiation on the blood-brain barrier and optimum time of chemotherapy. Int J Radiat Oncol Biol Phys. 1990;19:1507-10.

38. Cao Y, Tsien CI, Shen Z, Tatro DS, Ten Haken R, Kessler ML, et al. Use of magnetic resonance imaging to assess blood-brain/ blood-glioma barrier opening during conformal radiotherapy. J Clin Oncol. 2005;23:4127-36.

39. Fan $\mathrm{CH}$, Liu WL, Cao H, Wen C, Chen L, Jiang G. $\mathrm{O}^{6}$-methylguanine DNA methyltransferase as a promising target for the treatment of temozolomide-resistant gliomas. Cell Death Dis. 2013;4:e876.

40. Quinn JA, Jiang SX, Reardon DA, Desjardins A, Vredenburgh JJ, Rich JN, et al. Phase II trial of temozolomide plus o6benzylguanine in adults with recurrent, temozolomide-resistant malignant glioma. J Clin Oncol. 2009;27:1262-7.

41. Watson AJ, Sabharwal A, Thorncroft M, McGown G, Kerr R, Bojanic S, et al. Tumor $\mathrm{O}(6)$-methylguanine-DNA methyltransferase inactivation by oral lomeguatrib. Clin Cancer Res. 2010;16:743-9.

42. Rood BR, Zhang $\mathrm{H}$, Cogen PH. Intercellular heterogeneity of expression of the MGMT DNA repair gene in pediatric medulloblastoma. Neuro Oncol. 2004;6:200-7.

43. Ranson M, Middleton MR, Bridgewater J, Lee SM, Dawson M, Jowle D, et al. Lomeguatrib, a potent inhibitor of O6-alkylguanine-DNA-alkyltransferase: phase I safety, pharmacodynamic, and pharmacokinetic trial and evaluation in combination with temozolomide in patients with advanced solid tumors. Clin Cancer Res. 2006;12:1577-84. 